\title{
THE TWENTY-FIRST SUMMER MEETING OF THE AMERICAN MATHEMATICAL SOCIETY.
}

By invitation of Brown University, the twenty-first summer meeting of the Society was held at that institution on Tuesday and Wednesday, September 8-9, 1914, in connection with the celebration of the one hundred and fiftieth anniversary of the founding of the university. Two sessions were held on Tuesday and a morning session on Wednesday, the following fifty-two members being in attendance:

Professor R. C. Archibald, Dr. Ida Barney, Professor G. D. Birkhoff, Professor J. W. Bradshaw, Professor B. H. Camp, Dr. A. B. Chace, Dr. A. Cohen, Professor F. N. Cole, Dr. C. F. Craig, Mr. C. H. Currier, Mrs. E. B. Davis, Professor E. W. Davis, Professor S. C. Davisson, Professor W. P. Durfee, Professor L. P. Eisenhart, Mr. H. J. Ettlinger, Professor G. C. Evans, Professor H. B. Fine, Professor T. S. Fiske, Dr. W. C. Graustein, Professor C. N. Haskins, Professor E. R. Hedrick, Professor E. V. Huntington, Professor W. A. Hurwitz, Dr. Dunham Jackson, Dr. R. A. Johnson, Professor Edward Kasner, Professor C. J. Keyser, Mr. John McDonnell, Dr. J. K. Lamond, Professor H. P. Manning, Professor G. A. Miller, Professor G. D. Olds, Dr. F. W. Owens, Professor Alexander Pell, Professor Anna J. Pell, Professor A. D. Pitcher, Professor T. M. Putnam, Professor R. G. D. Richardson, Professor E. D. Roe, Jr., Mrs. E. D. Roe, Jr., Professor D. A. Rothrock, Dr. H. M. Sheffer, Professor H. E. Slaught, Professor E. R. Smith, Professor J. N. Van der Vries, Professor E. B. Van Vleck, Professor A. G. Webster, Professor J. H. M. Wedderburn, Professor H. S. White, Professor Ruth G. Wood, Professor J. W. Young.

The President of the Society, Professor E. B. Van Vleck, occupied the chair at the morning sessions; Vice-President L. P. Eisenhart presided at the Tuesday afternoon session. The Council announced the election of the following persons to membership in the Society: Mr. Lincoln K. Adkins, University of Minnesota; Dr. Lennie P. Copeland, Wellesley College; Mr. John W. Cromwell, Jr., Washington, D. C., High Schools; Professor Tsuruichi Hayashi, Tôhoku Imperial University, Sendai, Japan; Professor Claude I. Palmer, Armour 
Institute of Technology; Mr. George A. Pfeiffer, Columbia University; Mr. Paul R. Rider, Yale University; Dr. Alfred Rosenblatt, University of Cracow; Miss Caroline E. Seely, Columbia University. Eleven applications for membership in the Society were received.

In order that those wishing to do so may attend the winter meeting of the Chicago Section, the meeting of Section A of the American Association, and the annual meeting of the Society (at which President Van Vleck will deliver his presidential address), it was voted to suspend By-Law III to the extent of holding the annual meeting not later than January 5 , 1915. A committee, consisting of Professors M. W. Haskell, H. F. Blichfeldt, L. E. Dickson, O. D. Kellogg, and the Secretary, was appointed to arrange for holding the summer meeting at San Francisco in 1915.

It was decided to issue only a List of Officers and Members next year, instead of the complete Annual Register.

The first session, on Tuesday morning, opened with an address of welcome by Chancellor A. B. Chace of Brown University. The social programme included a tea in the John Carter Brown Library on Tuesday afternoon and luncheon in Rockefeller Hall on Wednesday, both given by Professor N. F. Davis. The University gave a dinner to the Society at the University Club on Tuesday evening, which was concluded by an address by President Faunce and an interesting account by Professor Carl Barus of the "Historical development of the modern theories of physics." A vote of thanks was tendered to the University and its officers for their generous hospitality. Wednesday afternoon was devoted to an excursion to Newport.

The following papers were presented at this meeting:

(1) Dr. F. M. Morgan: "A plane cubic Cremona transformation and its inverse."

(2) Professor L. P. Eisenhart: "Conjugate systems with equal tangential invariants and the transformation of Moutard."

(3) Professor C. E. Love: "Singular integral equations of the Volterra type."

(4) Professor O. E. Glenn: "Modular invariant processes."

(5) Professor L. E. Dickson: "Invariants, seminvariants and covariants of the ternary and quaternary quadratic form modulo $2 . "$ 
(6) Professor L. E. Dickson: "The points of inflexion of a plane cubic curve."

(7) Professor L. E. Dickson: "A fundamental set of modular invariants of the system of the binary cubic, quadratic, and linear form."

(8) Professor L. E. Dickson: "Invariants in the theory of numbers."

(9) Professor F. B. Wiley: "Proof of the finiteness of the modular covariants of a system of binary forms and cogredient points."

(10) Professor E. V. Huntington: "The theorem of rotation in elementary dynamics."

(11) Dr. R. D. BeEtle: "Congruences associated with a one-parameter family of curves."

(12) Professor G. C. Evans: "The non-homogeneous parabolic differential equation."

(13) Dr. R. A. Johnson: "The conic as a space element."

(14) Professor W. A. Hurwitz and Dr. L. L. Siluverman: "On the consistency and equivalence of certain definitions of summability."

(15) Professor Maxime Bôcher: "The method of successive approximations for linear differential systems."

(16) Professor Maxime Bôcher: "The smallest characteristic numbers in a certain exceptional case."

(17) Professor B. H. CAMP: "On the series obtained by term-wise integration."

(18) Professor G. A. Miller: "On the $\varphi$-subgroup of a group."

(19) Dr. T. E. Mason: "On functions transcendentally transcendental with respect to a given realm of rationality."

(20) Dr. T. E. Mason: "Mechanical device for testing Mersenne numbers for primes."

(21) Mr. H. S. Vandiver: "On Bernoulli's numbers, Fermat's quotient and last theorem."

(22) Professor L. C. KarPinski: "An early algorism."

(23) Professor H. S. White: "Triple-systems on 31 letters; a reconnoissance."

(24) Miss L. D. Cummings: "The trains for 42 non-congruent triple-systems on 15 elements."

(25) Professor J. H. M. Wedderburn: "On matrices whose coefficients are entire functions."

(26) Professor E. R. Sмiтh: "A problem in the fitting of polynomial curves to certain kinds of data." 
(27) Dr. H. R. Kingston: "Metric properties of nets of plane curves."

(28) Professor G. D. BIRKHoFf: "The iterated transformation of a plane into itself."

(29) Professor W. B. Fite: "Prime power groups in which every commutator of prime order is invariant."

(30) Professor Edward Kasner: "Transversality for double integrals in the calculus of variations and for contact transformations."

(31) Professor Edward Kasner: "The decomposition of conformal transformations into factors of period two."

(32) Professor R. G. D. Richardson: "A new boundary value problem for linear hyperbolic differential equations of the second order."

(33) Dr. Joseph Rosenbaum: "Mixed linear integral equations over a two-dimensional region."

(34) Professor D. C. Gillespie: "Cauchy's definition of a definite integral."

Dr. Kingston's paper was communicated to the Society by Professor Wilczynski. Dr. Rosenbaum was introduced by Professor Hurwitz. In the absence of the authors, Miss Cummings's paper was read by Professor White and the papers of Dr. Morgan, Professor Love, Professor Glenn, Professor Dickson, Professor Wiley, Dr. Beetle, Professor Bôcher, Dr. Mason, Mr. Vandiver, Professor Karpinski, Dr. Kingston, Professor Fite, and Professor Gillespie were read by title.

Abstracts of the papers follow below. The abstracts are numbered to correspond to the titles in the list above.

1. In this paper Dr. Morgan studies the properties of the plane cubic Cremona transformation $S=\left(\underset{y_{1}\left(x_{1}^{2}+y_{1}{ }^{2}\right)}{x} x_{1}\left(x_{1}+y_{1}^{2}+y_{1}\right) x_{1} y_{1} z_{1}\right)$ and its inverse $T=\left(\begin{array}{ccc}x_{1} & y_{1} \\ x y^{2} & y x^{2} & z\left(x^{2}+y^{2}\right)\end{array}\right)$. Both of these transformations are examples of an $\alpha_{i}$ coinciding with an $\alpha_{k}$. When written in non-homogeneous coordinates these transformations present a simple ruler and compass construction by means of which one may construct a cissoid, a four-leaved rose, a lemniscate, and other higher plane curves.

2. When the tangential coordinates of a surface $S$ are solutions of an equation of the form

$$
\frac{\partial^{2} \theta}{\partial u \partial v}+\frac{\partial \log \rho}{\partial v} \frac{\partial \theta}{\partial u}+\frac{\partial \log \rho}{\partial u} \frac{\partial \theta}{\partial v}=0
$$


the parametric coordinate system is said to have equal tangential invariants. Professor Eisenhart shows that there exist transformations of $S$ into surfaces $S_{1}$ such that the parametric system on $S_{1}$ is conjugate with equal tangential invariants, and moreover the lines joining corresponding points on $S$ and $S_{1}$ form a congruence whose developables meet these surfaces in the parametric curves. In this case the surfaces $S$ and $S_{1}$ are said to be in the relation of a transformation $\Omega$. The surfaces associate to $S$ and $S_{1}$ with the asymptotic lines of the former corresponding to the parametric conjugate systems on the latter can be so placed in space that they are the focal surfaces of a $W$-congruence. If $S$ and $S_{1}$ are in the relation of a transformation $\Omega$, the tangent planes to these surfaces at corresponding points are harmonic to the focal planes of the congruence of lines of intersection of these planes. Of particular interest is the case in which the latter congruence is normal. To this class belong the transformations of surfaces of Voss discussed by the author in the July number of the Transactions. The transformations of surfaces with isothermal spherical representation of their lines of curvature established in the ninth volume of the Transactions are transformations $\Omega$ and the only ones such that $S$ and $S_{1}$ are the two sheets of the envelope of a two-parameter family of spheres. If $S_{1}$ and $S_{2}$ are two transforms of $S$, there exist an infinity of surfaces $S^{\prime}$ each of which is in the relation of a transformation $\Omega$ with $S_{1}$ and $S_{2}$. Moreover, if $M, M_{1}, M_{2}$ are the corresponding points of $S, S_{1}, S_{2}$, the corresponding points of the surfaces $S^{\prime}$ lie on a conic in the plane of $M, M_{1}$, $M_{2}$; and the tangent planes to the surfaces $S^{\prime}$ pass through the point of intersection of the corresponding tangent planes to $S, S_{1}, S_{2}$.

The transformation of Moutard of equations of the type (1) plays an important rôle in the transformations $\Omega$, as also in the transformations $K$ of conjugate systems with equal point invariants announced by the author at the April meeting of the Society. The paper will appear in the Palermo Rendiconti.

3. Professor Love's paper proves the existence of a continuous solution $\varphi(x)$ of the singular Volterra equation of the second kind

$$
\varphi(x)=f(x)+\int_{x}^{\infty} K(x, t) \varphi(t) d t
$$


under various hypotheses concerning the functions $f(x)$ and $K(x, t)$. In each case the behavior of $\varphi(x)$ for large values of $x$ is also considered. The paper will appear in the October number of the Transactions.

4. Under linear transformations on the variables $x_{i}(i=1$, $\cdots, m)$ whose coefficients are positive residues of a prime number $p$ the functions $x_{i}^{p^{t}}(i=1, \cdots, m)$ are cogredient to the variables. Professor Glenn proves this and shows that it leads to the fact that the polar operator

$$
x_{1}{ }^{p^{t}} \frac{\partial}{\partial x_{1}}+\cdots+x_{m}{ }^{p^{t}} \frac{\partial}{\partial x_{m}},
$$

applied to any covariant of an algebraic $m$-ary form $f$, gives a formal modular covariant of $f$. Modular transvectants are also defined by means of such cogredient expressions, and their properties discussed. Application is made to the derivation of an irreducible system of formal modular concomitants of a linear form, consisting of two invariants and six covariants (cf. Dickson, Madison Colloquium Lectures, page 40).

Since the transformation on the $x$ 's induces a similar group of transformations on the coefficients $a_{0}, a_{1}, \cdots$, we have a dual theory of the modular Aronhold operators

$$
a_{0}{ }^{p^{t}} \frac{\partial}{\partial a_{0}}+\cdots+a_{\mu} p^{t} \frac{\partial}{\partial a_{\mu}} \text {. }
$$

5. The object of the first paper by Professor Dickson is to provide an improved method for finding the leading coefficient of a modular covariant of a form or forms in three or more variables. For the case of a ternary quadratic form modulo 2, the method employed in the Madison Colloquium Lectures, pages $77-79$, is sufficiently brief, but is not as expeditious as the new method, especially in more difficult problems. The paper will appear in the Bulletin.

6. In the second paper by Professor Dickson, an elementary and largely novel exposition is given of the classic theory of the inflexion points of a plane cubic curve without singular points. Then the Galois group of the equation $X$ for the abscissas of the nine inflexion points is determined for special cubic curves as well as for the curve in whose equation the 
coefficients are independent variables. The knowledge of the group and its properties is applied to a complete analysis of the solution by radicals of the equation $X$. The paper will appear in the December number of the Annals of Mathematics.

7. The third paper by Professor Dickson shows how simple and remarkably effective is the theory of modular invariants based upon the separation of equivalent sets of ground forms into classes (cf. Madison Colloquium Lectures), by treating the simultaneous modular invariants of the binary cubic, quadratic, and linear form. The paper will appear in the Quarterly Journal of Mathematics.

8. A. Hurwitz* introduced the concept of formal invariants of binary forms (whose coefficients are independent variables) under the group of all binary linear transformations with integral coefficients taken modulo $p$, a prime. Aside from the cases $\dagger$ of quadratic and cubic binary forms, and a theorem by Miss Sanderson $\ddagger$ connecting the subject with modular invariants, no progress has been made towards a general theory. A remarkably simple and effective method of constructing formal invariants is given in the fourth paper by Professor Dickson. In the case of a single form $f$ in $n$ variables, the method consists in forming the symmetric functions of certain like powers of the values of $f$ at the real points in modular space. The power used is such that $f$ has the same value at $\left(x_{1}, \cdots, x_{n}\right)$ as for $\left(\rho x_{1}, \cdots, \rho x_{n}\right)$, where $\rho$ is any integer. In the case of several forms $f_{i}$, we employ the symmetric functions of $\phi\left(v_{1}, v_{2}, \cdots\right)$, in which $v_{1}, v_{2}, \cdots$ range over the sets of values of powers of $f_{1}, f_{2}, \cdots$ at the real points, while $\phi$ is any polynomial with integral coefficients. We may also employ the points whose coordinates are in a Galois field of order $p^{k}$. From the special cases examined, it appears that the method furnishes a fundamental system of formal invariants. There is an immediate extension to the case of seminvariants and to the general case of invariants under any modular group. The paper will appear in the Transactions.

* Archiv der Mathematik und Physik (3), vol. 5 (1903), p. 25.

$\dagger$ Dickson, The Madison Colloquium Lectures, pp. 41-54.

$\ddagger$ Transactions, vol. 14 (1913), p. 490. 
9. The question of the finiteness of the modular covariants of a system of forms and cogredient points arises naturally at this time in view of the fact that Professor Dickson has proved (Transactions, 1913) the finiteness of the modular covariants of a system of forms without cogredient points and Professor Krathwohl secured in his Chicago dissertation (American Journal, October, 1914) a fundamental set of modular covariants of two cogredient binary points. In the present paper Professor Wiley secures the theorem:

The set of all modular covariants of any system of forms in $x_{01}, x_{02}$ and the cogredient points $x_{k 1}, x_{k 2}(k=1, \cdots, n)$ is finite in the sense that they are all rational integral functions, with integral coefficients taken modulo $p$, of a finite number of covariants of the set.

This paper will appear in the Transactions.

10. In the theory of the plane motion of a rigid body, the equation $F r=(W / g) k^{2} d \omega / d t$, where $d \omega / d t$ is the angular acceleration of the body at the instant considered, plays an important rôle. In this equation, $F r$ is the sum of the moments of the external forces and $k$ the radius of gyration of the body about a suitably chosen point $Q$. The question at once arises, what is meant by a suitably chosen point in this case. All the books agree that the equation is true if $Q$ is fixed in the body and fixed in space, or if $Q$ coincides with the center of mass. Many books state that the equation is true also when $Q$ is the instantaneous center of rotation, and some that it is true when $Q$ is any point whatever. In view of these discrepancies in the current elementary textbooks, it seems worth while to review the facts concerning this important equation. In order to do this most effectively, Professor Huntington states and proves two distinct theorems, one for the case in which $Q$ is fixed in the body, the second for the case in which $Q$ is fixed in the plane.

I. If $Q$ is a point fixed in the body, then the equation $F r=(W / g) k^{2} d \omega / d t$ is true: (1) when $Q$ is fixed in the plane, (2) when $Q$ is moving with constant velocity in a straight line, (3) when $Q$ is the center of mass, (4) when $Q$ has an acceleration whose direction at the instant in question passes through the center of mass; and in no other cases. In particular, it is not in general legitimate to "take moments about the instantaneous center." 
II. If $Q$ is fixed in space, $F r=(W / g) d(c \bar{v}) / d t+(W / g) k_{0}{ }^{2} d \omega / d t$, where $\mathrm{Fr}$ is the sum of the moments of the external forces about the point $Q$ at the instant in question, $c \bar{v}$ is the moment of the velocity of the center of mass about $Q$ at that instant, and $k_{0}$ is the radius of gyration about the center of mass.

These two theorems are of course implicitly contained in any standard treatise on the subject; but they do not seem to be explicitly stated in this form in any of the elementary textbooks. The proofs are here given in a very direct and simple form.

11. With a one-parameter family of curves may be associated a number of rectilinear congruences. The congruence formed by the tangents to the curves has been very extensively studied, and there are many theorems concerning the relations between the properties of this congruence and those of the curves and of the surface on which they lie. The other congruences naturally associated with a one-parameter family of curves have received little attention. In this paper, Dr. Beetle considers some of these other congruences, giving particular attention to those formed by the principal normals and binormals of the curves.

In connection with certain properties of the congruences, it is of interest to consider those surfaces on which the asymptotic lines in one system, or both, are geodesic parallels. It is shown that the determination of all such surfaces requires the solution of a rather complicated partial differential equation of the fourth order. Several characteristic properties of these surfaces are found.

12. Professor Evans considers the non-homogeneous partial differential equation $\partial u / \partial t-\partial^{2} u / \partial x^{2}=f(x, t)$ and the function which is its principal solution, when the proper derivatives exist, with the object of generalizing the conditions on $f(x, t)$ necessary for the existence of these derivatives. According to the nature of the parabolic equation, such conditions should be asymmetrical in character. It is proved that those derivatives exist at the point $\left(x_{1}, t_{1}\right)$ provided that $f(x, t)$ is finite and integrable in a suitably defined region, and at $\left(x_{1}, t_{1}\right)$ is continuous and satisfies the condition $\left|f(x, t)-f\left(x_{1}, t\right)\right|$ $\leqq N\left|x-x_{1}\right|^{\tau}$, where $N$ and $\tau$ are positive numbers, not zero.

13. Dr. Johnson uses as coordinates of the conic in three- 
dimensional space the elements of the discriminant of its tangential equation; in other words, a conic is represented by ten homogeneous coordinates which are elements of a vanishing four-rowed symmetrical determinant. Thus there is a one-to-one correspondence between the conics of space and points of a quartic hypersurface in space of nine dimensions. The equations, in terms of these coordinates, which correspond to various projective relations between conics and other figures, such as points, lines, planes, quadrics, are developed for numerous simple cases. After a brief consideration of the relation of apolarity between quadrics, a detailed discussion is given of the possible linear systems of conics.

14. In the present paper Professor Hurwitz and Dr. Silverman, on the ground of previous work by Silverman, Toeplitz, and Schur, establish a correspondence between certain functions $f(z)$ and certain definitions of summability of a divergent sequence. If $f(z)$ is analytic within and on the boundary of the circle $C$ of radius $\frac{1}{2}$ about the point $\frac{1}{2}$, the corresponding definition is a generalization of convergence, $i$. e., correctly evaluates any convergent sequence. All such definitions are consistent, i. e., if two of them evaluate a certain sequence, they give it the same value. Two definitions are equivalent, i. e., evaluate exactly the same sequences, provided the corresponding functions have the same zeros with the same multiplicities in $C$. Further properties of the definitions in question are derived; the equivalence of the Cesàro and Hölder definitions of the same order appears as an incidental result. The question of equivalence of different definitions has previously received some slight attention; it is believed that the question of consistency is here studied for the first time.

15. The results partially announced by Professor Bôcher at the Cambridge International Congress in 1912 concerning the scope of the method of successive approximations when applied in a very general manner to ordinary linear differential systems are here developed in detail.

16. Professor Bôcher's second paper appeared in full in the October Bulletin.

17. It has long been known that, if a series is uniformly convergent, the series obtained by integrating it term-wise 
is also uniformly convergent. In recent years more general sufficient conditions for term-wise integrability have been discovered, and finally, by Vitali and W. H. Young, conditions which are both necessary and sufficient. If a series which is not uniformly convergent be integrable term-wise over a variable interval, the new series may not be uniformly convergent, but it has certain other characteristics which may be classified according to which of these sufficient conditions the original series satisfies. Furthermore, successive integrations over the same interval lead to progressively simpler series.

In Professor Camp's paper several general theorems dealing with this subject are developed. A particular result, capable of being stated concisely, is that, if a series may be integrated term-wise in the variable interval $(a, x)$, then the new series may be integrated term-wise, even after being multiplied through by any function of $x$ which is absolutely integrable in Lebesgue's sense.

18. All the operators of any group $G$ can be divided into two categories having no common operator by putting into one category $(A)$ all those operators of $G$ which can occur in one of the possible sets of independent generators and into the other category $(B)$ those which do not have this property. The category $(B)$ is a subgroup of $G$, and this subgroup was called by $G$. Frattini the $\varphi$-subgroup of $G$. It is always the direct product of its Sylow subgroups and hence it is always solvable. The $\varphi$-subgroup of $G$ may also be defined as the cross-cut of all the possible maximal subgroups of $G$. A simple example of a $\varphi$-subgroup is the subgroup of order $p^{m-1}$ in the cyclic group of order $p^{m}, p$ being any prime number.

Professor Miller developed a number of theorems relating to the $\varphi$-subgroups. In particular, he proved that the order of the $\varphi$-subgroup of any abelian group of order $p^{m}$ is $p^{m-a}$, whenever $p^{\alpha}$ is the order of the subgroup generated by all of the operators of order $p$. A necessary and sufficient condition that the $\varphi$-subgroup of an abelian group be identity is that all the Sylow subgroups of this abelian group are of type $(1,1,1, \cdots)$. If a group can be represented as a primitive substitution group its $\varphi$-subgroup is identity; in particular, the $\varphi$-subgroup of every simple group of composite order is identity. 
19. Dr. Mason finds conditions that first and second order linear difference and $q$-difference equations and Riccati difference and $q$-difference equations have only solutions that are transcendentally transcendental with respect to a general realm of rationality which includes the coefficients. These conditions are expressed in the possibility of certain auxiliary equations having solutions algebraic in the realm of rationality considered. The results for these equations in the realm of rationality $x$ are to be found in papers by Tietze, Stridsberg, and a previous paper by Dr. Mason.

20. Lucas has given a method of testing Mersenne numbers for primes, in which he uses multiplication in the binary system of numeration. Dr. Mason shows how multiplication in this system can be reduced to counting correspondences, and suggests a mechanical device to do the counting and recording.

21. Mr. Vandiver obtains some general theorems respecting Fermat's quotient and Bernoulli's numbers and in particular the following relation:

$$
\frac{\left(m^{a}-1\right) h_{a}}{a} \equiv \sum_{n=1}^{p-1} y_{n} n^{a-1} \quad(\bmod p),
$$

where $1<a<p-1,1<m<p$ ( $p$ a prime),

$$
\begin{gathered}
y_{n} \equiv-\frac{n}{p} \quad(\bmod m), \quad m>y_{n} \geqq 0, \\
h_{2 a}=(-1)^{a-1} B_{a}, \quad h_{2 a+1}=0, \\
B_{1}=\frac{1}{6}, \quad B_{2}=\frac{1}{30}, \quad \text { etc. }
\end{gathered}
$$

By means of this theorem it is then shown that Kummer's criteria in reference to Fermat's last theorem may be replaced by the following conditions:

$$
\sum_{n=1}^{p-1} y_{n} n^{p-2} t^{n} \equiv 0 \quad(\bmod p) \quad(m=2,3, \cdots, p-1) .
$$

(See notation used in Crelle, volume 144, page 314.) A number of otber transformations of the Kummer congruences are also given. 
22. The Latin treatises on the Hindu art of reckoning which are earlier than the fourteenth century are sufficiently rare so that the addition even of anonymous works to the available literature is desirable. An algorism which bears internal evidence that it was one of the very early treatises on this subject has been found by Professor Karpinski in the British Museum. Royal MS .15 B IX contains the algorism in question; this manuscript is dated by the experts of the museum as of the end of the twelfth or beginning of the thirteenth century. A somewhat similar work, directly related to this algorism, is found in the Egerton MS 2261, fol. 225 $227^{\mathrm{b}}$, and a second copy in Codex Paris, 10252, fol. $68^{\mathrm{a}}-70^{\mathrm{b}}$. The copy in Paris, written 1476, was found by Björnbo and is included in his list of medieval mathematical works which is preserved in Stockholm. A study is made of all three of the manuscripts.

This paper will appear in the Bibliotheca Mathematica.

23. The number of different triad systems on $n$ letters is known only for the small values of $n$. Reiss proved that at least one system exists for every value of $n=6 t+1$ or $n=6 t+3$. E. H. Moore proved that at least two incongruent systems exist for every such $n$ above 13. Zulauf showed two distinct systems for 13. Miss Cummings has distinguished 24 systems for $n=15$ without exhausting the possibilities. In the present paper, from one simple theorem and certain data not yet published, Professor White demonstrates the existence of a very large number, above $10^{13}$, of systems of a particular kind on 31 letters.

24. Miss Cummings has applied to triple-systems on $\mathbf{1 5}$ elements the method for determining the non-congruency of triple-systems given by Professor H. S. White in the Transactions of January, 1913. The paper shows the 203 distinct types of trains which occur in 42 non-congruent systems $\Delta_{15}$.

25. In this paper, Professor Wedderburn extends the methods ordinarily used in reducing to its normal form a matrix whose coefficients are polynomials in a single variable so as to make these methods applicable when polynomials are replaced by entire functions or, more generally, by functions holomorphic in a simply connected region. 
26. If to a series of values of $x$ which are in an arithmetical progression there corresponds a series of observed values of $y$, and if it is desired to determine by the method of least squares or by the method of moments the coefficients of the polynomial

$$
y=a_{0}+a_{1} x+a_{2} x^{2}+\ldots+a_{k} x^{k}
$$

which give the best relation between $x$ and $y$, the problem can be simplified by evaluating the determinant

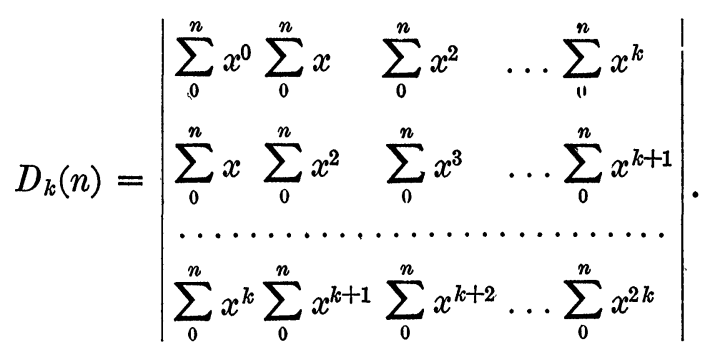

By separating the determinant into two factors Professor Smith finds the roots of $D_{k}(n)$ and consequently its factors. It follows then that when $n$ is less than $k+1, D_{k}(n)$ vanishes, when $n=k+1, D_{k}(n)$ is the square of a simple Vandermondian, and finally when $n$ is greater than $k+1$,

$$
D_{k}(n)=C_{k} n^{v=k-1} \prod_{v=0}^{v-1}(n-k+v)^{v+1}(n+k-v)^{v+1},
$$

where $C_{k}$ is a constant for any value of $k$. Having found this value for the determinant, simple expressions are obtained for the coefficients $a_{0}, a_{1}, a_{2}, \ldots, a_{k}$ which depend only on the observed values of $y$ and on the number of the observations.

27. In a memoir entitled "One-parameter families and nets of plane curves,"* Wilczynski has discussed the projective differential properties of nets of plane curves, by means of a completely integrable system of three partial differential equations of the second order. In Dr. Kingston's paper the foundation is laid for a study of the metric differential properties of such nets. In order to accomplish this, it becomes necessary to consider, besides the coefficients of the partial differential equations used by Wilczynski, the coefficients $E$, $F$, and $G$ of the square of the element of arc when this is ex-

* Transactions, vol. 12 (1911), pp. 473-510. 
pressed in terms of the parameters of the net curves as coordinates. All of these quantities taken together, which must moreover satisfy certain relations, determine a net uniquely except for a motion combined with a reflection. The application of the general theory to the particular cases of orthogonal and isothermal nets gives rise to interesting results.

28. The iterated transformation of a plane into itself leads to hitherto untreated problems in point set theory which stand in vital relation to certain dynamical questions. The problem considered by Professor Birkhoff is that of characterizing as far as possible the sequence of positions taken by an arbitrary point of the plane under repetition of the transformation and its inverse. Some of the results obtained run parallel to those given by him in an earlier paper.*

29. Professor Fite's paper concerns itself with those prime power groups in which every commutator of prime order is invariant. It is shown that if $G$ is of order $p^{m}$ ( $p$ an odd prime) and any cogredient has a cyclic central, the second central must contain a commutator of order $p$ that is not invariant in $G$. In fact, if any cogredient contains a non-invariant operation of order $p$, the second central must contain a commutator of order $p$ that is not invariant in $G$. Of various other results obtained the two following may be mentioned: (1) If every operation of the second central whose order does not exceed $p^{r}$ is invariant in $G$, every operation of $G$ whose order does not exceed $p^{r}$ is invariant. (2) If the commutator subgroup is cyclic and the $(k-1)$ th central is of class $k_{1}, G$ must be of class $k$, where $k \leqq 3 k_{1}$.

30. In connection with the minimizing of double integrals, Kneser's theory of transversality gives rise to a correspondence between the surface elements and the lineal elements at any point of space. This correspondence is not entirely arbitrary in character. Professor Kasner obtains a simple geometric property which furnishes a criterion as to when a given correspondence can be regarded as a transversality correspondence. In particular the only linear correspondences satisfying the test are polarities.

*Bulletin Soc. Math. de France, vol. 40 (1912), pp. 305-323. 
The general result obtained is also applied to the theory of infinitesimal contact transformations, and indirectly to the transversality correspondences associated with simple integrals in space. Certain connections between simple and double integrals are thus derived.

31. The conformal transformations discussed in Professor Kasner's second paper are assumed to be regular in the neighborhood of the origin. They are thus expressed by power series in $x+i y$ or $x-i y$ without absolute term. It is shown that there are two types of transformations of period two: the first, in $x+i y$, are called conformal involutions; and the second, in $x-i y$, are identified with symmetries (in the general sense of Schwarz). The main results of the paper are criteria as to when a given conformal transformation can be regarded as the product of either two involutions, or two symmetries. The classes of transformations thus obtained are very large, only a finite number of restrictions being imposed on the coefficients of the corresponding power series. As in the author's earlier results in conformal invariants, distinctions arise according to the rationality or irrationality of certain angles determined by the coefficients.

32. For linear hyperbolic differential equations of the second order in two variables, the boundary value problem ordinarily considered is that of finding a solution $u(x, y)$ which on a piece of a curve has prescribed values for $u$ and $\partial u / \partial n$. The simplest case of the new problem studied consists in determining whether there exist parameter values $\lambda$ of the equation

$$
\begin{gathered}
\left(p u_{x}\right)_{x}-\left(q u_{y}\right)_{y}+\lambda u=0 \\
{\left[p(x, y) \geqq k_{1}>0, \quad q(x, y) \geqq k_{2}>0\right]}
\end{gathered}
$$

such that the corresponding solutions vanish on the boundary of a region $a_{1} \leqq x \leqq a_{2}, b_{1} \leqq y \leqq b_{2}$. Closely associated with this problem are new problems in linear equations and in quadratic forms each with an infinite number of variables, as well as with $n$ variables where $n$ is allowed to increase indefinitely. Professor Richardson has shown that there is a denumerable infinity of parameter values $\lambda$ of the equation (1) for which there are solutions of the type sought. This set of values has limiting points at both positive and negative 
infinity as well as at one or more finite points. Under restrictions similar to those usually imposed, a function $f(x, y)$ may be developed in a series of solutions with Fourier coefficients.

33. Following out methods previously used by Professor W. A. Hurwitz in discussing mixed linear integral equations in one dimension, Dr. Rosenbaum in this paper establishes similar results for the case of two dimensions. The unknown function appears under integral signs operating over a plane region and over curves, and the values of the unknown function at special points also appear in the equation. The adjoint system of equations now involves as unknowns one function of two variables, several functions of one variable, and several constants. The notions of resolvent system, orthogonalization of principal solutions, and pseudo-resolvent system, receive similar generalizations.

34. The Riemann definition of the definite integral of a bounded function involves the values of the function at arbitrary points of the sub-intervals of a scheme of subdivision, while the Cauchy definition involves the values only at ends of sub-intervals. It is obvious that if the Riemann integral exists the Cauchy integral will exist; it is not immediately evident whether the converse is true or false. Professor Gillespie proves in this note that the two definitions are equivalent.

F. N. Cole, Secretary.

\section{INFINITE REGIONS IN GEOMETRY.}

BY PROFESSOR EDWIN BIDWELL WILSON.

(Read before the American Mathematical Society, February 28, 1914.)

THE recent contribution by Professor Bôcher on "The infinite regions of various geometries"* puts me in mind of some ideas which I have long held on this subject and which I desire to offer to readers of his article.

The points which he most desires to make are:

$1^{\circ}$, that when we are dealing with other geometries than

* This Bulletin, vol. 20, pp. 185-200, January, 1914. 